\title{
Clinical sabbatical aims to beef up trial-management skills
}

Clinical psychiatrist Barbara Gracious had a month to spare over the summer while transitioning to her new job at Ohio State University in Columbus. But, instead of jetting off to a vacation spot, she packed her bags for Bethesda, Maryland, home of the US National Institutes of Health (NIH) Clinical Center, to go on sabbatical.

This past summer, Gracious, together with three other researchers, was part of a pilot training program in clinical trial management. The investigators took courses in introductory protocol writing, patient tracking and safety management, among other issues. They also met with program officers at the NIH and made contacts at the US Food and Drug Administration (FDA) to better understand regulatory issues associated with clinical research.

Beyond the coursework, Gracious says the experience provided the perfect networking opportunity. "I was able to meet with other researchers who will be co-investigators or consultants on my planned work... and provide input or share laboratory techniques to better the science for our patients," she says.

Clinical Center director John Gallin says the program was set up to foster these kinds of connections, as well as to help researchers deal with the management and bureaucratic aspects of running a clinical trial. "There's a real need to demystify the federal bureaucracy and regulatory components that have become demanding for investigators," he says.

Giselle Sholler, a pediatric oncologist from the University of Vermont in Burlington who was also part of the pilot program, was no novice to clinical grant writing, having already netted four NIH clinical trials over the past four years. But as her work expands to include more collaborations with other institutions, she wanted more formal training in how to most effectively budget and use resources for larger trials.

Reflecting on her sabbatical experience thus far (she expects to complete the program next June), Sholler thinks the experience gave her a better idea of what to expect from government regulators. "That's not something we're taught in medical school," she says.

Beyond training to help ensure that medical research programs are safe, ethical and efficient, the Clinical Center's sabbatical program also aims to familiarize researchers with the business side of running clinical trials, including planning budgets and negotiating with subcontractors. James Bost, a biostatistician who manages large clinical trials at the University of Pittsburgh says this knowledge is crucial to the success of a trial.

"You get to the point where you can't keep everything in house," says Bost, who is not affiliated with the sabbatical program.

After the successful pilot program, the Clinical Center is now accepting applications year round for new participants from anywhere in the world. Two of the pilot participants were from Russia, and Gallin says the program "has appeal and applicability to both US and international investigators."

Roxanne Palmer

\section{The knee bone's connected to the... titanium foam?}

By some estimates, more than two million bone grafts take place worldwide each year. The best possible graft material is autologous bone taken from the patient-usually shaved from the side of the pelvis. But researchers are busy developing advanced titanium foams for certain circumstances in which artificial grafts work best.

"You might choose an [artificial substance] in a person who has a cancer, if you're worried that bone may not grow" when exposed to radiation therapy, says Michael Yaszemski, a professor of orthopedic surgery and biomedical engineering at the Mayo Clinic in Rochester, Minnesota.

Engineered bone grafts have been evolving in sophistication for decades. One of the oldest types uses ceramics derived from calcium phosphate, which mimics the mineral structure of bone. Bone cells can readily recognize and bond to this material.

Metal implants, generally made from titanium or tantalum, were solid at first, but the second generation was refined by making them more porous, to allow bone growth throughout the implant and reduce the risk of infection.

The newest type of metal bone implants rely on titaniumbased foam. One such creation, called TiFoam, is made by impregnating a form made of polyurethane foam with titanium powder. The structure hardens into a metallic mesh, after which the polyurethane is washed away. TiFoam was developed by Peter Quadbeck, an engineer who works at the Dresden branch of the Fraunhofer Institute, a German applied-materials research organization.
"The main difference is a lower stiffness, as compared to solid titanium, and a higher ductility, as compared to brittle ceramic material," says Quadbeck.

Quadbeck started developing TiFoam in 2007, and the project was completed in late September. Although TiFoam has not been tested in humans yet, Quadbeck says it performed well in yet-to-be-published animal tests where it was used to replace intervertebral discs in sheep. Recent studies, unaffiliated with Quadbeck's group, have successfully tested titanium foam implants in rabbit femurs and the tibias of rats ( $\mathrm{J}$. Biomed. Mater. Res. B. Appl. Biomater. 92, 479-488, 2010; J. Biomed. Mater. Res. B. Appl. Biomater. 94, 64-71, 2010). These studies showed greater bone ingrowth when a foam implant was used compared to a denser metal mesh.

The possible drawback to titanium foam is the one common to all artificial bone implants: any kind of metal implant is there to stay, unlike other kinds of new bone grafts in development that contain biodegradable scaffolds.

Even if it does become commercialized, TiFoam wouldn't be the first metal foam implant on the market. It faces competition from, for example, Plivio Pore, an implant designed specifically for use in spinal fusion surgery, manufactured by the Pennsylvania-based medical device company Synthes. But Quadbeck says his foam is more porous than the current products on the market, allowing for greater bone ingrowth in larger implants.

Roxanne Palmer 